\title{
Hexanuclear Complex of Platinum(II) with Cleavage Product of Cystamine Dihydrochloride by $\beta$-Mercaptoethylamine
}

\author{
Asmat N. Azizova, Dilqam B. Tagiyev, Shmid G. Gasimov, Khudayar I. Gasanov \\ The Institute of Catalysis and Inorganic Chemistry Named after Acad. M. F. Nagiyev of the National Academy of \\ Sciences Azerbaijan, Baku, Azerbaijan \\ Email:mmanafov@gmail.com
}

Received 5 October 2015; accepted 9 January 2016; published 12 January 2016

Copyright $(\subset 2016$ by authors and Scientific Research Publishing Inc.

This work is licensed under the Creative Commons Attribution International License (CC BY).

http://creativecommons.org/licenses/by/4.0/

(c) (i) Open Access

\begin{abstract}
Six-nuclear complexes of Platinum(II) with cleavage of a cystamine dihydrochloride by $\beta$-mercaptoethylamine with composition $\left[\mathrm{Pt}_{6}\left(\mathrm{H}_{2} \mathrm{NCH}_{2} \mathrm{CH}_{2} \mathrm{~S}\right)_{8}\right] \mathrm{Cl}_{4} \cdot 5 \mathrm{H}_{2} \mathrm{O}$ had been syntesized for the first time. Structure of the complex had been installed together with IKS- and XRD analysis. It has been established that each atom of platinum is surrounded by two atoms of sulfur and nitrogen due to bidentate coordination of a ligand. Only two of the six platinum atoms are on the same plane; other four atoms are in the different crossing planes. Four platinum atoms form adurable metal chelate cycles, which collapse over temperature $260^{\circ} \mathrm{C}$. The results of IR spectroscopy confirmed with the RS-analysis.
\end{abstract}

\section{Keywords}

Mercaptoamine, Ligands, Platinum, Six-Nuclear Complexes, Synthesis

\section{Introduction}

In the study of the complexation of Platinum(II) and Palladium(II) compounds with cystamine dihydrochloride, it has been shown that sulfur-sulfur bond of cystamine doesn't only undergo cleavage in non-aqueous media. The onium complexes had been obtained in the non-aqueous medium where cysteamine acted as the cation containing protonated amine group. In other cases, the reaction in an aqueous medium cleavage of sulfur-sulfur occurs with the formation of mono- or bidentate coordinated mercamine complexes. Depending on the $\mathrm{pH}$ and temperature of the medium it had been obtained complexes with various types of coordination mercamine [1].

The behavior of cystamine dihydrochloride has been studied in this paper in an alkaline medium $(\mathrm{pH}=9-10)$ 
in the presence of Platinum(II) and has been showed under these conditions in cystamine cleavage of sulfursulfur bond occurring with subsequent formation of the deprotonated $\beta$-mercaptoethylamine. Under the coordination of cleavage product with Platinum(II) atoms, it has been obtained hexanuclear complex following this composition- $\left[\mathrm{Pt}_{6}\left(\mathrm{H}_{2} \mathrm{NCH}_{2} \mathrm{CH}_{2} \mathrm{~S}\right)_{8}\right] \mathrm{Cl}_{4} \cdot 5 \mathrm{H}_{2} \mathrm{O}$.

The paper contains the results of the synthesis, IR spectroscopy and X-ray diffraction study of the compound.

\section{Experimental}

\subsection{Methods and Apparatus}

IR spectra of the complex-[ $\left[\mathrm{Pt}_{6}\left(\mathrm{H}_{2} \mathrm{NCH}_{2} \mathrm{CH}_{2} \mathrm{~S}\right)_{8}\right] \mathrm{Cl}_{4} \cdot 5 \mathrm{H}_{2} \mathrm{O}$, and IR spectra cystamine dihydrochloride, $\beta$-mercaptoethylamine hydrochloride had been measured in the spectral range $200-500 \mathrm{~cm}^{-1}$ on Fourier transform infrared (FTIR) spectrometer IFS-113V in the form of suspensions fluorinated oils and vaseline between the windows of $\mathrm{KBr}$ and polyethylene.

\subsection{Synthesis of $\left[\mathrm{Pt}_{6}\left(\mathrm{H}_{2} \mathrm{NCH}_{2} \mathrm{CH}_{2} \mathrm{~S}\right)_{8}\right] \mathrm{Cl}_{4} \cdot 5 \mathrm{H}_{2} \mathrm{O}$}

From $\mathrm{H}_{2}\left[\mathrm{PtCl}_{6}\right] \cdot 6 \mathrm{H}_{2} \mathrm{O}$-by reduction with hydrazinedihydrochloride was getting- $\mathrm{H}_{2}\left[\mathrm{PtCl}_{4}\right]$ and slowly added in portions of $40 \%$ solution cystamine dihydrochloride. The resulting precipitate is being filtered off and prepared aqueous suspension. To this suspension with stirring added $25 \%$ aqueous ammonia solution until $\mathrm{pH}=10$ medium and precipitate dissolved. The resulting clear solution was filtered and left at room temperature for slow crystallization. Five days later, yellow color single crystals precipitated from the solution, which is filtered, washed with ethanol, then ether and dried in vacuum to constant weight.

For complex $\mathrm{Pt}_{6} \mathrm{C}_{16} \mathrm{H}_{58} \mathrm{~N}_{8} \mathrm{~S}_{8} \mathrm{O}_{5} \mathrm{Cl}_{4}$;

Found, \%: Pt-58.32; Cl-7.21; N-5.70; C-9.42; H-2.74;

Calculated, \%: Pt-58.20; Cl-7.05; N-5.56; C-9.55; H-2.88.

\section{Results and Discussion}

Comparison of the IR spectra cystamine dihydrochloride, $\beta$-mercaptoethylamine hydrochloride and complex leads to the conclusion that in the complex with platinum involves $\beta$-mercaptoethylamine, but not cystamine.

The fact of bidentatny coordination of $\beta$-mercaptoethylamine with forming a five-membered metallacycle chelate on nitrogen atom of the amino group and thiolate sulfur occupying bridged position follows from the data of the IR spectra of the complex $\left[\mathrm{Pt}_{6}\left(\mathrm{H}_{2} \mathrm{NCH}_{2} \mathrm{CH}_{2} \mathrm{~S}\right)_{8}\right] \mathrm{Cl}_{4} \cdot 5 \mathrm{H}_{2} \mathrm{O} \quad v_{P t-S}^{\text {bridg }}=286 \mathrm{CM}^{-1}, v_{\mathrm{NH}_{2}}^{\text {coord }}=3170 \mathrm{CM}^{-1}$, $v_{P t-N}=378 \mathrm{CM}^{-1}$.

The obtained results are in good agreement with literature data [2] on the IR spectra of mononuclear platinum complex composition $\mathrm{PtL}_{2}$, where L-bidentate coordinated $\beta$-mercaptoethylamine, as well as with our earlier [1] for the binuclear complex- $\left.\mathrm{Pt}_{2}\left(\mathrm{~S}_{2} \mathrm{CH}_{2} \mathrm{CH}_{2} \mathrm{NH}_{3}\right)_{2} \mathrm{Cl}_{4}\right]$, for which bridging position of thiolate atom of sulfur was established by $\mathrm{X}$-ray analysis.

$\mathrm{X}$-ray analysis of the faceted monocrystals of the complex showed that the triclinic cell parameters: $a=9.671$ (4), $b=11,278$ (5), $c=12,490$ (5) $\AA, \alpha=64.85$ (3), $\beta=104.61$ (4), $\gamma=92.44$ (4) ${ }^{0}, \mathrm{Z}=1$ formula unit $\mathrm{Pt}_{6} \mathrm{~S}_{8}$ $\mathrm{Cl}_{4} \mathrm{~N}_{8} \mathrm{C}_{16} \mathrm{H}_{48} \cdot 5 \mathrm{H}_{2} \mathrm{O}$, lim. facet. $\bar{P}_{1}$.

The experimental material comprised 5292 independent nonzero Paterson fractions and a series of Fourier synthesis, including the difference. Hydrogen atoms are localized partially from the difference Fourier synthesis.

It should be noted that in the elucidation of the structure had been set statically disordered arrangement of one of the two non-equivalent crystallographic chlorine atoms on the two positions of $\mathrm{Cl}(2) \mathrm{Cl}(3)$ and one of the oxygen atoms of water molecules in the position $\mathrm{O}(3)$ with a multiplicity of 0.5 .

For all non-hydrogen atoms anisotropic refinement had been conducted. Final $\mathrm{R}=0.045$.

The basic of structure is constituted by hexanuclear complex cations $\left[\mathrm{Pt}_{6}\left(\mathrm{NH}_{2} \mathrm{CH}_{2} \mathrm{CH}_{2} \mathrm{~S}\right)_{8}\right]^{4+}$ (Figure 1), Clions and water molecules. Six platinum atoms are located at the vertices of an octahedron, with four of them being coordinated 8 deprotonated molecules $\beta$-mercaptoethylamine with cis arrangement of the atoms of nitrogen and thiolate sulfur atoms. Each atom Pt (2) and Pt (3) closes the two five-membered metallacycle PtSCCN.

Coordinated sphere of atoms Pt (1) consist of four bridging sulfur atom. All platinum atoms have squareplanar coordination with small tetrahedral distortion. 


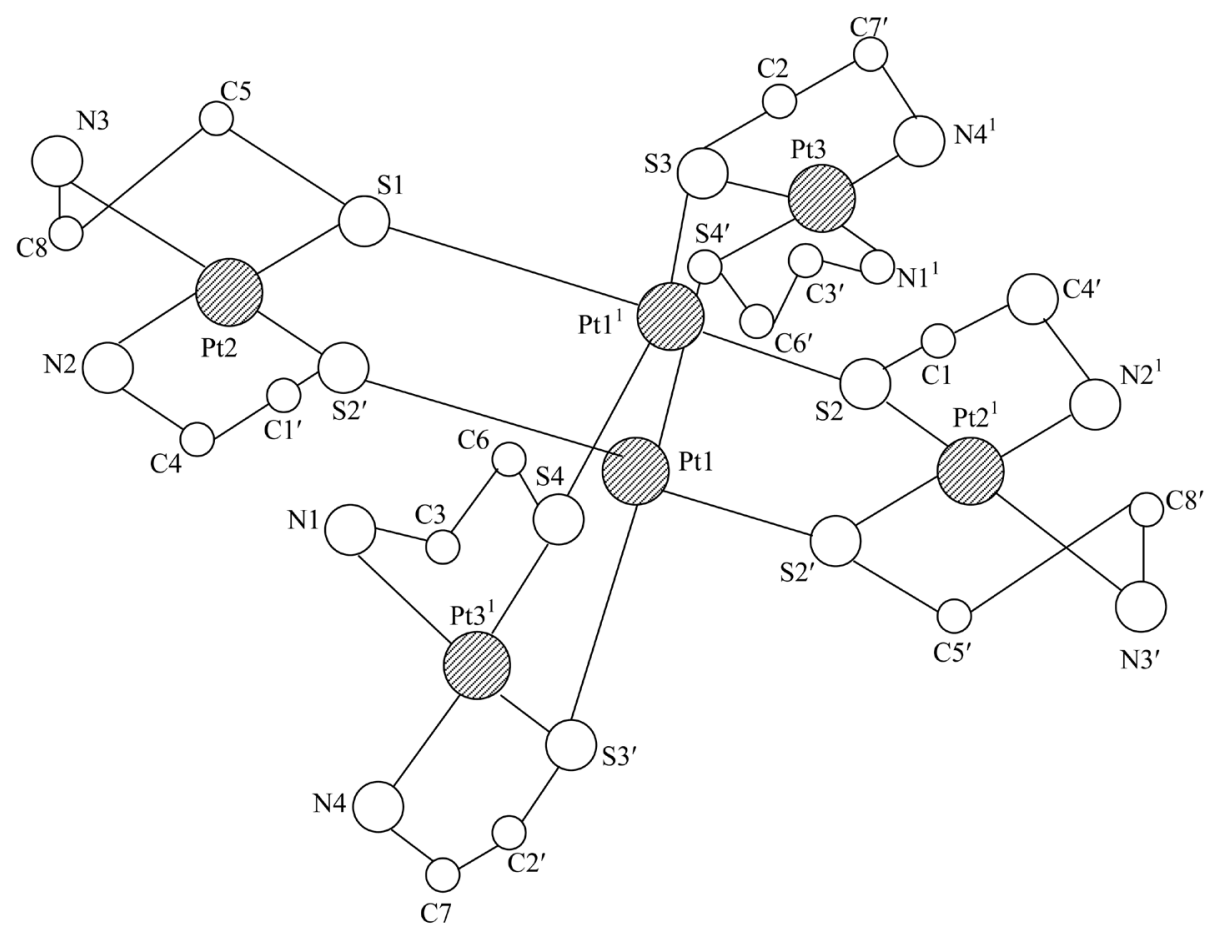

Figure 1. Hexanuclear platinum complex with beta mercaptoethylamine.

Sulfur atoms formed around Pt (1) substantially undistorted flat square with spacing Pt (1)-S in the range of 2.330 (2) - 2.339 (2) $\AA$, with an average value of $2.334 \AA$. Angles at the atom Pt (1) slightly deviate from the ideal square-planar geometry $\{89.68(7)-80.47(7)\}$. Minor deviations of the atoms from the mean plane of focal angle $\mathrm{PtS}_{4}$ for S- $( \pm 0.002)$, for the Pt (1)-0.04 $\AA$.

As opposed to Pt (1), in the metal chelate of Pt (2) and Pt (3) the coordination unit $\mathrm{PtS}_{2} \mathrm{~N}_{2}$ have cis-structure and experience a significant distortion. Bond lengths of Pt (2)-S, and Pt (3)-S 2.274 (2) - 2.280 (2) A , with an average value of $2.277 \AA$ are reduced comparing to Pt (1)-S approximately about $0.06 \AA$.

Pt-N distances are in the range 2.076 (8) - 2.099 (6) $\AA$, with an average value of $2.087 \AA$. There is a large variation in the valence angles at atoms Pt (2) and Pt (3) $\{85.7$ (2) - 94.3 (2) $\}$ as compared to those of Pt (1).

Deviations of atoms from the average plane of the coordination unit $\mathrm{PtN}_{2} \mathrm{~S}_{2}$ make for $\mathrm{S}$ and $\mathrm{N}$ at $\mathrm{Pt}$ (2)$( \pm 0.005 \AA$ ), at Pt (3)- $( \pm 0.011 \AA)$. Atoms Pt (2) and Pt (3) pushed out from the plane to $0.376 \AA$ and $0.359 \AA$, respectively.

As previously studied platinum complexes (II) with Pt-N and Pt-S bonds values vary in the range 1.978 (3) 2.059 (2) $\AA$ [3]-[5] and 2.282 (3) - 2.342 (2) $\AA$ [6]-[8], respectively.

Observed hardening Pt-S linkages in metal chelates due, apparently, to the presence of sulfur atoms, having $\pi$-accenting properties and the ability of the second donor nitrogen atoms locate in trans-position to the sulfur atom, to transfer excess electronic density to the corresponding $\mathrm{AO}$ of the metal.

Tetrahedral distortion of coordination node in metal chelates (the dihedral angles between the planes $\mathrm{Pt}(2) \mathrm{S}$ (1) N (3)/Pt (2) S (2)N (2) and Pt (3) S (3) N (7)/Pt (3) S (4) N (1) equal to $38.8^{\circ}$ and $44.5^{\circ}$, respectively), is probably due to steric strain resulting from the features of its structure-cis-bond reduction Pt-S, shortening distances S-S and N-S by approximately 0.5 - $0.6 \AA$ compared with the sum of the van der Waals radiuses of sulfur and nitrogen atoms.

In centrosymmetric hexanuclear complex there are two mutually perpendicular metallacycle passing through atoms Pt (1) and $\mathrm{Pt}\left(1^{1}\right)$.

The shortest distance between the platinum atoms is 3.201 (1) $\AA$, which indicates the absence of metal-metal interactions. Each cycle consists of four atoms Pt and four $S$ atoms and hasa chair conformation. Atoms $S$ (3), $S$ (4), and Pt (1) lie substantially in a plane, shift make for S (3)-( $\pm 0.005 \AA)$, for S (4)- $( \pm 0.003 \AA)$. Atoms of Pt (3) are deployed on opposite sides of the plane so that the angle between the planes 2Pt (1) 2S (3) 2 S (4) (1) and S 
(3) S (4) Pt (3) is equal to $51.5^{\circ}$. For the second plane 2Pt (1) 2S (1) 2S (2) (2) deviations of atoms constitute for $\mathrm{S}(1)-( \pm 0.010 \AA)$ and S (2)-( $\pm 0.006 \AA)$. Atoms Pt (2) arealso deployed in opposite directions, the angle between the planes (2) and S (1) S (2) (2) Pt (2) is equal to 69.40 and between the planes 1 and is $289.7^{\circ}$.

In metal chelates PtSCCN bond lengths of S-C, C-C and C-N have values in the range of 1.83 (1) - 1.85 (1), 1.48 (2) - 1.52 (2) и 1.47 (2) - 1.52 (2) $\AA$, respectively. These distances might be compare to those, for example, nickel trinuclear complex [Ni( $\left.\mathrm{Ni}\left(\mathrm{NH}_{2} \mathrm{CH}_{2} \mathrm{CH}_{2} \mathrm{~S}\right)_{2}\right]_{2} \mathrm{Cl}_{2}$ [9]-[11].

Valence angles between pointed bonds deflected from tetrahedral not more than \pm 20 and are in the range of $107.4^{\circ}$ (5) $-111.4^{\circ}$ (6). All molecules $\beta$-mercaptoethylamine have twist conformation, the torsion angles of SCCN make $52.7^{\circ}-57.6^{\circ}[12]-[15]$.

Interaction between hexanuclear complexes are carried by using chlorine ions and water molecules. Chloride ions involved in hydrogen bonds with the nitrogen atoms of amino groups and oxygen of the water molecules as proton acceptor at distances Cl... N (0) 3.02 (3) - 3.54 (2) Å. Furthermore hydrogen bonds are formed between the water molecules and also the water molecules and the amino groups of the ligand, wherein the oxygen atoms act both as donor and proton acceptor. O... O distances (N) are in the range of 2.99 (1) - 3.15 (2) A.. Existence of rather short distances of N-H... Cl-3.02 (3) - 3.33 (1) $\AA$ allows to believe that the structure is stabilized partly by means of hydrogen bonds.

\section{Conclusion}

Thus, for the first time, $\mathrm{X}$-ray analysis revealed the existence of six-core complex of Platinum(II) non-clustered type. The basic of structure is constituted by hexanuclear complex cations $\left[\mathrm{Pt}_{6}\left(\mathrm{NH}_{2} \mathrm{CH}_{2} \mathrm{CH}_{2} \mathrm{~S}\right)_{8}\right]^{4+}$, Cl-ions and water molecules. Six platinum atoms are located at the vertices of an octahedron, with four of them being coordinated 8 deprotonated molecules $\beta$-mercaptoethylamine with cis arrangement of the atoms of nitrogen and thiolate sulfur atoms. Each atom of Pt (2) and Pt (3) is closing the two of five-membered metallacycle PtSCCN.. Observed hardening Pt-S linkages in metal chelates due, apparently, to the presence of sulfur atoms, having $\pi$-accenting properties and the ability of the second donor nitrogen atoms locate in trans-position to the sulfur atom, to transfer excess electronic density to the corresponding $\mathrm{AO}$ of the metal.

\section{References}

[1] Hasanov, H.I. (2003) Synthesis, Structure and Properties of Coordination Compounds of Platinum(II) and Palladium(II) with a Biologically Active Ligands. Doctoral Thesis, Baku.

[2] Sheldrick, G.M. (2008) A Short History of SHELX. Acta Crystallographica Section A, 64, 112-122. http://dx.doi.org/10.1107/S0108767307043930

[3] Schlapfer, C.W. and Nakamoto, K. (1972) Far-Infrared Spectra of Mercaptoalkylamine Complexes with $\mathrm{Ni}^{\mathrm{II}}$ and $\mathrm{Pd}^{\mathrm{II}}$. Inorganica Chimica Acta, 6, 177-181. http://dx.doi.org/10.1016/S0020-1693(00)91780-8

[4] Farrugia, L.J. (1999) WinGX Suite for Small-Molecule Single-Crystal Crystallography. Journal of Applied Crystallography, 32, 837-838. http://dx.doi.org/10.1107/S0021889899006020

[5] Hiller, A.C. and Nolan, S.P. (2002) Palladium/Nucleophilic Carbene Catalysts for Cross-Coupling Reactions. Platinum Metals Review, 46, 50-64.

[6] Sharupin, V.V., Sengurin, V.S. and Pakusina, A.P. (2010) Reactions Triphenylbenzylphosphonium Chloride and Potassium Tetrachloroplatinate Tetrafenilstibonite with Dimethyl Sulfoxide. Russian Journal of General Chemistry, 80, 1753-1757. http://dx.doi.org/10.1134/S1070363210090057

[7] Canty, A.J., Minchin, N.J., Skelton, B.W. and White, A.H. (1986) Interaction of Palladium(II) Acetate with Substituted Pyridines, including a Cyclometallation Reaction and the Structure of $\left[\mathrm{Pd}\left\{\right.\right.$ meso-[(py) $\left.\left.\mathrm{PhMeC}_{2} \mathrm{C}_{5} \mathrm{H}_{3} \mathrm{~N}\right\}\left(\mathrm{O}_{2} \mathrm{CMe}\right)\right]$ $\left[\mathrm{O}_{2} \mathrm{CMe}\right] \cdot 3 \mathrm{H}_{2} \mathrm{O}$. Journal of the Chemical Society, Dalton Transactions, No. 10, 2205-2210. http://dx.doi.org/10.1039/dt9860002205

[8] Nakanishi, J. and Matsumoto, K. (1991) Structures of Benzenethiolato Complexes of Palladium(II) and Platinum(II). Acta Crystallographica, 47, 2073. http://dx.doi.org/10.1107/s0108270191004675

[9] Campbell, M.G., Powers, D.C., Raynaud, J., Graham, M.J., Xie, P., Lee, E. and Ritter, T. (2011) Synthesis and Structure of Solution-Stable One-Dimensional Palladium Wires. Nature Chemistry, 3, 949-953. http://dx.doi.org/10.1038/nchem.1197

[10] Sheldrick, G.M. (2002) SHELX: 30 Years of Open Source Experience. Acta Crystallographica Section A, 58, 254. http://dx.doi.org/10.1107/s0108767302095181 
[11] Mahmudov, K.T., Hasanov, X.I., Maharramov, A.M., Azizova, A.N., Ragimov, K.Q., Askerov, R.K., Kopylovich, M.N., Ma, Z. and Pombeiro, A.J.L. (2013) A Hexanuclear Metallacrown Palladium(II) Cluster Derived from 2-Mercaptoethanol. Inorganic Chemistry Communications, 29, 37-39. http://dx.doi.org/10.1016/j.inoche.2012.12.012

[12] Stromnoka, T.A., Monakhov, K.Yu. and Cdmpora, J. (2007) Synthesis and Solution Behavior of the Trinuclear Palladium(II) Unsaturated Carboxylate Complexes Triangle-Pd ${ }_{3}\left[\mu-\mathrm{O}_{2} \mathrm{CC}\left(\mathrm{R}^{\prime}\right)=\mathrm{CHMe}\right]_{6}\left(\mathrm{R}^{\prime}=\mathrm{Me}, \mathrm{H}\right)$ : X-Ray Structure of Palladium(II) Tiglate (R' = Me). Inorganica Chimica Acta, 360, 4111-4116. http://dx.doi.org/10.1016/j.ica.2007.05.058

[13] Zaitsey, V.G., Shabashov, D. and Daugulis, O. (2005) Highly Regioselective Arylation of sp3 C-H Bonds Catalyzed by Palladium Acetate. Journal of the American Chemical Society, 127, 1315.

[14] Bakmutov, V.I., Berry, J.F., Ibragimov, S. and Murillo, C.A. (2005) Non-Trivial Behavior of Palladium(II) Acetate. Dalton Transactions, No. 11, 1989-1992. http://dx.doi.org/10.1039/b502122g

[15] Wei, C.H. and Dahl, L.F. (1970) Structural Characterization of Crystalline Tetrakis(2-Aminoethanethiol)Trinickel(II) Chloride, $\left[\mathrm{Ni}\left\{\mathrm{Ni}\left(\mathrm{NH}_{2} \mathrm{CH}_{2} \mathrm{CH}_{2} \mathrm{~S}\right)_{2}\right\}_{2}\right] \mathrm{Cl}_{2}$. New Basic Trinuclear Metal System Containing Nickel(II)-Nickel(II) Interactions. Inorganic Chemistry, 9, 1878-1887. http://dx.doi.org/10.1021/ic50090a018 